\title{
Síndrome de Gilles de la Tourette associada ao transtor- no de déficit de atenção com hiperatividade: resposta clínica satisfatória a inibidor seletivo da recaptura de serotonina e metilfenidato
}

Tourette syndrome associated with attention-deficit hyperactivity disorder: satisfactory clinical response to selective serotonin reuptake inhibitor and methylphenidate

Roberta Benitez Freitas Passos', José Ramón Rodriguez Arras López²

\section{RESUMO}

\section{Palavras-chave}

Síndrome de Tourette, transtorno do déficit de atenção com hiperatividade, inibidores de captação de serotonina, metilfenidato.

\section{Keywords}

Tourette syndrome, attention-deficit disorder with hyperactivity, serotonin uptake inhibitors, methylphenidate.

\begin{abstract}
A Síndrome de Gilles de la Tourette (SGT), caracterizada pela presença de tiques motores e vocais, apresenta elevada associação com transtorno obsessivo-compulsivo (TOC) e transtorno de déficit de atenção com hiperatividade (TDAH). Essas condições frequentemente causam mais prejuízo aos pacientes do que os tiques, propriamente. Relata-se o caso clínico de um paciente com SGT e comorbidade com TDAH e TOC. O tratamento com inibidor seletivo de recaptura de serotonina (ISRS) e metilfenidato promoveu melhora significativa dos sintomas de TDAH, sintomas compulsivos e tiques.
\end{abstract}

\section{ABSTRACT}

Tourette Syndrome (TS), characterized by motor and vocal tics, is often associated with obsessive compulsive disorder (OCD) and attention-deficit hyperactivity disorder (ADHD). These associated conditions frequently cause more impairment in patients than tics themselves. We report the case of TS with comorbid ADHD and OCD. Treatment with selective serotonin reuptake inhibitor and methylphenidate, led to significantly improvement of ADHD symptoms, compulsive symptoms and tics.

\section{INTRODUÇÃO}

A síndrome de Gilles de la Tourette é um transtorno neuropsiquiátrico, diagnosticado, geralmente, pela primeira vez na infância ou adolescência, caracterizado por tiques motores associados a tiques vocais, por pelo menos um ano'. Inicialmente considerada uma síndrome muito rara, atualmente se estima que sua prevalência situe-se entre 4 e 6 casos/1.000 crianças $^{2}$. Afeta aproximadamente três vezes mais o sexo masculino ${ }^{2}$.

A presença de comorbidade é extremamente elevada, estando em torno de $80 \%{ }^{3}$. Estudos epidemiológicos revelam que mais da metade dos pacientes com Gilles de la Tourette apresenta transtorno de déficit de atenção com hipe-

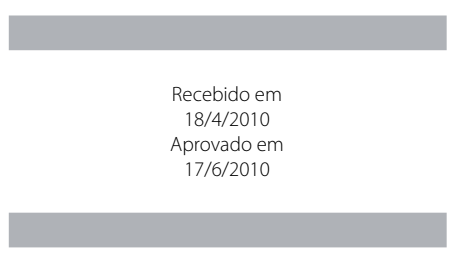

1 Universidade do Estado do Rio de Janeiro (UERJ), Instituto de Medicina Social (IMS), Departamento de Epidemiologia, Coordenação de Aperfeiçoamento de Pessoal de Nível Superior (Capes).

2 Universidade Federal do Estado do Rio de Janeiro (Unirio), Escola de Medicina e Cirurgia (EMC), Hospital Universitário Gaffrée e Guinle (HUGG), Serviço e Disciplina de Psiquiatria. 
ratividade associado ${ }^{4}$. Esse mesmo percentual de pacientes exibe sintomas obsessivo-compulsivos, e apenas um terço dos casos preenche critérios para transtorno obsessivo-compulsivo ${ }^{5}$.

Essas condições associadas à SGT frequentemente causam mais prejuízo aos pacientes do que propriamente os tiques ${ }^{6}$. O tratamento farmacológico da SGT visa à minimização do impacto social e acadêmico, e não necessariamente à supressão dos tiques. Tradicionalmente, usam-se neurolépticos (como o haloperidol e a pimozida,) antipsicóticos atípicos ou agonistas 2 (como a clonidina, por exemplo)?

Como o TDAH é altamente prejudicial ao desempenho acadêmico, seu tratamento é imperativo ${ }^{7}$. Relatos de casos e observações clínicas sugeriram, entretanto, que o metilfenidato pode exacerbar os tiques em alguns indivíduos com Tourette ${ }^{8}$. Contudo, as evidências advindas de ensaios clínicos randomizados não confirmam essa hipótese ${ }^{9}$.

Neste estudo, será descrito o caso de um paciente com SGT manejado com sucesso com um inibidor da recaptura de serotonina.

\section{DESCRIÇÃO DO CASO CLÍNICO}

A publicação do caso foi autorizada pela mãe do paciente, tendo sido o termo de consentimento anexado ao prontuário hospitalar.

A., masculino, 12 anos, brasileiro, negro, natural do Rio de Janeiro, cursando a $4^{a}$ série do ensino fundamental.

Nascido a termo, de parto cesáreo em virtude de eclampsia, apresentou desenvolvimento neuropsicomotor sem alterações. A mãe refere que, quando bebê, ele era calmo. Contudo, desde os 3 anos de idade, passou a apresentar comportamento hiperativo. Na escola, ficava muito agitado, batendo e mordendo os colegas. Em casa, ele gostava de assistir a desenhos animados na televisão, mas "levantava o tempo todo, não parava quieto".

Aos 7 anos, na 1a série do ensino fundamental, as professoras passaram a relatar manifestações de déficit de atenção: "ele vivia desligado" e não interagia. A família buscou atendimento neuropsiquiátrico em outra instituição. Foram prescritos risperidona e ácido valproico. A mãe não percebeu melhora. A seguir, introduziu-se carbamazepina, "que o deixava bobo". Foi reprovado na escola.

No ano seguinte (aos 8 anos), continuava com a medicação prévia e foi associada fluoxetina, sem sucesso. Apresentou ganho de peso significativo, pois "comia compulsivamente". Ainda nessa época, passou a exibir tique motor, "piscar demais os olhos" e algum tempo depois a apresentar tiques vocais manifestados como "pigarros", que é como a mãe chamava os ruídos que o paciente emitia como se estivesse "limpando ou aliviando uma comichão na garganta". Essas vocalizações ocorriam numa frequência semelhante e, por vezes, acompanhando o abrir e fechar rítmico das pálpebras. A mãe informa que alguns meses depois iniciaram as compulsões: quando caminhava, "parecia que estava medindo tudo, colocava um pé atrás do outro". Além disso, "se abrisse a porta, tinha que fazer isso várias vezes" e, ao assistir a desenhos animados, passou a repetir a fala de todos os personagens, de todos os exames. Foi possível observar alguns desses fenômenos no primeiro exame.

Foi encaminhado ao Serviço de Psiquiatria do Hospital Universitário Gaffrée e Guinle (HUGG) para avaliação diagnóstica e conduta terapêutica (aos 9 anos, na $2^{\text {a }}$ série). Na ocasião, estava em uso de carbamazepina 100 mg/dia, ácido valproico 1,5 g/dia, risperidona $1 \mathrm{mg} /$ dia e fluoxetina $20 \mathrm{mg} / \mathrm{dia}$. Durante a consulta, apresentava hiperatividade, mostrando-se bastante inquieto, andando pela sala, manuseando os objetos à sua frente, como o carimbo do médico. Apresentava prejuízo significativo no desempenho escolar e social. O paciente exibia comportamento impulsivo. Relata que os colegas de escola chamavam-no de esquisito e, às vezes, ele "não aguentava e batia neles". Segundo a mãe, as brigas eram semanais e ela encontrava-se bastante preocupada, pois A. estudava com bolsa em uma escola particular. Ela temia que ele perdesse o benefício por causa do mau comportamento e do baixo rendimento escolar.

Foram diagnosticados transtorno de Tourette, transtorno obsessivo-compulsivo e transtorno de déficit de atenção com hiperatividade, tipo combinado, segundo os critérios diagnósticos do DSM-IV-TR. A conduta consistiu em aumentar a fluoxetina para $30 \mathrm{mg} /$ dia. Houve melhora rápida da irritabilidade (duas semanas) e gradual das compulsões e tiques (dois meses), confirmada pela professora. A risperidona foi, então, suspensa e iniciou-se a redução gradual da dose de carbamazepina e ácido valproico, até sua retirada completa. Contudo, a hiperatividade e a impulsividade eventuais persistiam com prejuízo escolar. Iniciou-se, então, metilfenidato $10 \mathrm{mg} /$ dia pela manhã, que era seu turno na escola. Segundo a mãe, após aproximadamente sete dias a concentração dele aumentou, assim como a tranquilidade. A professora informou que A. passou a copiar a matéria no caderno e a prestar atenção às aulas.

A mãe relatou que com o tratamento A. passou a comer menos e "as repetições diminuíram". Houve redução "no piscar dos olhos", vocalizações e "mania de pisar com um pé na frente do outro". Cessaram os comentários sobre os tiques na escola. "Pararam de chamá-lo de esquisito", diz a mãe.

Com a introdução do metilfenidato, apresentou redução acentuada da hiperatividade, refletindo-se em melhora da coordenação e psicomotricidade, a qual pode ser observada por meio da mudança na caligrafia do paciente. Além disso, o desenvolvimento da atenção voluntária levou à melhora significativa em seu desempenho escolar. No ano em que iniciou o tratamento no HUGG, ficou entre os 10 melhores 
alunos da turma. O controle dos tiques motores e vocais possibilitou melhor relacionamento interpessoal. Após a suspensão da risperidona, carbamazepina e ácido valproico e início de uso do metilfenidato, houve redução de $10 \mathrm{~kg}$ de massa corporal em um ano, atingindo a faixa de peso esperada para sua altura.

O paciente é acompanhado no serviço há dois anos, não tendo sido necessário aumento de dose das medicações nesse período.

\section{DISCUSSÃO}

Trata-se de um caso característico de SGT. Os sintomas de TDAH precedem os tiques motores, os quais, usualmente, se manifestam antes das vocalizações. Os sintomas compulsivos surgiram por último. A coprolalia, tida como típica do transtorno, ocorre em menos de um terço dos $\operatorname{casos}^{10}$, não sendo observada no caso relatado.

Muitas vezes, torna-se difícil diferenciar TOC de sintomas obsessivo-compulsivos integrantes da SGT. Contudo, é sugerido que rituais de lavagem e checagem são mais frequentes em pacientes com TOC, enquanto pacientes com SGT exibem mais comumente compulsões de tocar, contar, piscar, sendo, dessa forma, vistas por muitos como tiques complexos ${ }^{11}$.

No caso descrito, o paciente não apresentou piora dos tiques com o uso do metilfenidato, beneficiando-se da meIhora no quadro de TDAH. Além disso, a fluoxetina levou à redução não apenas dos sintomas compulsivos, mas também, possivelmente, dos tiques. Uma vez que o paciente continuou sendo acompanhado no serviço, mantendo a melhora observada há dois anos, acredita-se que esse fato não seja apenas uma oscilação espontânea da intensidade dos sintomas, decorrente da evolução da doença, mas sim efeito da medicação.

Embora alguns ensaios clínicos tenham evidenciado ausência de efeito ou pouco efeito da fluoxetina nos tiques ${ }^{12,13}$, relatos de casos, bem como a nossa experiência clínica, vêm demonstrando bons resultados. Uma hipótese neuroquímica para isso seria a deficiência de serotonina e seus metabólitos, observada em alguns estudos ${ }^{14}$.

Kurlan et al. ${ }^{13}$ avaliaram 11 crianças com SGT em uso de haloperidol ou clonidina, ao qual foi adicionado, randomicamente, fluoxetina (20-40 mg/dia) ou placebo ${ }^{13}$. Os pacientes relataram melhora na intensidade e capacidade de supressão dos tiques com a adição de fluoxetina, sugerindo algum efeito potencial dos ISRS no tratamento de tiques.

Convém ressaltar que o ensaio clínico conduzido por Scahill envolveu apenas 14 pacientes e a dose de fluoxetina foi estabelecida em 20 mg/dia, sem possibilidade de aumento ${ }^{12}$. Dessa forma, o baixo poder do estudo (em virtude do pequeno tamanho amostral), bem como a baixa dose admi- nistrada de fluoxetina, pode ter sido responsável pela ausência de resposta dos tiques.

\section{CONCLUSÃO}

$O$ paciente descrito foi manejado satisfatoriamente apenas com ISRS e metilfenidato, sem necessidade de uso de bloqueadores dopaminérgicos para controle dos tiques da SGT.

Se confirmada a eficácia dos ISRS no manejo dos tiques, estes constituirão uma alternativa terapêutica vantajosa, dado o perfil de eventos adversos mais favorável, possibilitando melhor qualidade de vida dos pacientes.

\section{DECLARAÇÃO DE CONFLITOS DE INTERESSE}

Não há conflitos de interesse associados à publicação deste caso clínico.

\section{REFERÊNCIAS}

1. American Psychiatric Association. Diagnostic and Statistical Manual of Mental Disorders. 4. ed. Text revision. Washington: American Psychiatric Association; 2000.

2. Robertson MM. The prevalence and epidemiology of Gilles de la Tourette syndrome. Part 1 : the epidemiological and prevalence studies. J Psychosom Res. 2008;65(5):461-72.

3. Spencer T, Biederman J, Harding M, O'Donnell D, Wilens T, Faraone S, et al. Disentangling the overlap between Tourette's disorder and ADHD. J Child Psychol Psychiatry. 1998;39(7):1037-44.

4. Roessner V, Becker A, Banaschewski T, Freeman RD, Rothenberger A. Developmental psychopathology of children and adolescents with Tourette syndrome-impact of ADHD. Eur Child Adolesc Psychiatry. 2007;16(Suppl 1):24-35.

5. Eapen V, Fox-Hiley P, Banerjee S, Robertson M. Clinical features and associated psychopathology in a Tourette syndrome cohort. Acta Neurol Scand. 2004;109(4):255-60.

6. Jankovic J. Tourette's syndrome. N Engl J Med. 2001;345(16):1184-92.

7. Scahill L, Erenberg G, Berlin Jr CM, Budman C, Coffey BJ, Jankovic J, et al. Contemporary assessment and pharmacotherapy of Tourette syndrome. NeuroRx. 2006;3(2):192-206.

8. Lowe TL, Cohen DJ, Detlor J, Kremenitzer MW, Shaywitz BA. Stimulant medications precipitate Tourette's syndrome. JAMA. 1982;247(8):1168-9.

9. Bloch MH, Panza KE, Landeros-Weisenberger A, Leckman JF. Meta-analysis: treatment of attention-deficit/hyperactivity disorder in children with comorbid tic disorders. J Am Acad Child Adolesc Psychiatry. 2009;48(9):884-93.

10. Bruun RD, Budman CL. The natural history of Tourette syndrome. Adv Neurol. 1992:58:1-6.

11. Holzer JC, Goodman WK, MCDougle CJ, Baer L, Boyarsky BK, Leckman JF, et al. Obsessivecompulsive disorder with and without a chronic tic disorder. a comparison of symptoms in 70 patients. Br J Psychiatry. 1994;164(4):469-73.

12. Scahill L, Riddle MA, King RA, Hardin MT, Rasmusson A, Makuch RW, et al. Fluoxetine has no marked effect on tic symptoms in patients with Tourette's syndrome: a double-blind placebo-controlled study. J Child Adolesc Psychopharmacol. 1997;7(2):75-85.

13. Kurlan R, Como PG, Deeley C, McDermott M, McDermott MP. A pilot controlled study of fluoxetine for obsessive-compulsive symptoms in children with Tourette's syndrome. Clin Neuropharmacol. 1993;16(2):167-72.

14. Singer HS. Neurobiology of Tourette syndrome. Neurol Clin. 1997;15(2):357-79. 